\title{
Wave propagation for a reduced model of blood coagula- tion
}

\author{
Nicolas Ratto ${ }^{1, *}$, Martine Marion ${ }^{1}$, and Vitaly Volpert ${ }^{1,2,3}$ \\ ${ }^{1}$ Institute Camille Jordan, UMR 5208 CNRS, University Lyon 1, Villeurbanne 69622, France \\ ${ }^{2}$ INRIA, Université de Lyon, Université Lyon 1, Institute Camille Jordan, Villeurbanne 69200, France \\ ${ }^{3}$ Peoples' Friendship University of Russia (RUDN University), Moscow 117198, Russian Federation
}

\begin{abstract}
Reduced mathematical models of blood coagulation can properly describe Thrombin Generation Test (TGT). Clot growth occurs as a reactiondiffusion wave, and the reduced model studied in this work describes this behaviour, preserving the differences observed between healthy and hemophilia subjects in the TGT.
\end{abstract}

\section{The coagulation process}

Blood coagulation is a complex process involving biochemical reactions in plasma initiated at the damaged wall and leading to the formation of fibrin clot that stops bleeding. Thrombin is the central enzyme of the coagulation cascade produced at the initiation and amplification stages of the coagulation reactions. Thrombin generation test (TGT) is one of the most widely used integral laboratory tests of blood coagulation sensitive to vast variety of coagulation disorders [1-4]. It consists in measurement of thrombin concentration in time during in vitro coagulation of a plasma sample. In spite of the complexity of blood coagulation kinetics including dozens of substances and reactions between them $[5,6]$, thrombin generation curves (TGC) are quite simple bell-shaped curves.

Detailed mathematical models of TGT contain dozens of ODEs and many parameters that can hardly be determined for individual patients [7], and the application of such models encounters certain difficulties. In this work, we consider a reduced model of blood coagulation derived from a more complete model [8-10]:

$$
\begin{gathered}
\frac{d u_{10}}{d t}=\left(k_{1}+k_{2} T+k_{3} T^{2}\right)\left(v_{10}^{0}-u_{10}\right)-k_{4} u_{10}, \\
\frac{d T}{d t}=\left(k_{5} u_{10}+k_{6} T+k_{7} T^{2}+k_{8} T^{3}\right) P-k_{9} T, \\
\frac{d P}{d t}=-\left(k_{5} u_{10}+k_{6} T+k_{7} T^{2}+k_{8} T^{3}\right) P,
\end{gathered}
$$

where $u_{10}$ is the activated factor $X a, T$ is the thrombin concentration, and $P$ the prothrombin concentration. This model can reproduce the TGT for both healthy and hemophilia subjects, and the dependence of thrombin generation curves on the concentrations of blood factors

\footnotetext{
*e-mail: nicolas.ratto@ec-lyon.fr
} 
[11]. Let us note that the value of parameter $k_{1}$ characterizes the initiation of the reaction at the wall, and $k_{5}$ the initial thrombin production. Parameters $k_{2}, k_{3}, k_{6}, k_{7}$, and $k_{8}$ correspond to the self-accelerating thrombin production due to a positive feedback loop involving activated factor $X a$. The simplification of the model based on the approximation of detailed equilibrium for fast reactions allows the reduction of the complete system of equations to the system of three equations with a polynomial dependence on thrombin concentration [11, 12]. Finally, parameters $k_{4}$ and $k_{9}$ show the action of the anticoagulating factors.

It is known that clot growth occurs as a reaction-diffusion wave [8, 13-15]. The goal of this work is to study this process with model (1)-(3) taking into account the spatial distribution of the involved concentration. In this case, the model becomes as follows:

$$
\frac{\partial u}{\partial t}=D \frac{\partial^{2} u}{\partial x^{2}}+F(u)
$$

Here $u=(X a, T, P)$, and $F$ is the vector of reaction rates defined in (1)-(3). The diffusion matrix $D$ is a diagonal matrix with positive diagonal elements.

\section{Dynamics of clot growth}

\subsection{Thrombin generation curves}

Thrombin generation curves for healthy subjects and hemophilia patients [11] were fitted by system (1)-(3). Each individual can be characterized by the values of parameters $k_{1}, \ldots, k_{9}$. In the space of three most sensitive parameters $k_{2}, k_{6}$ and $k_{9}$, healthy and hemophilia subjects are clearly separated (Figure 1, left).
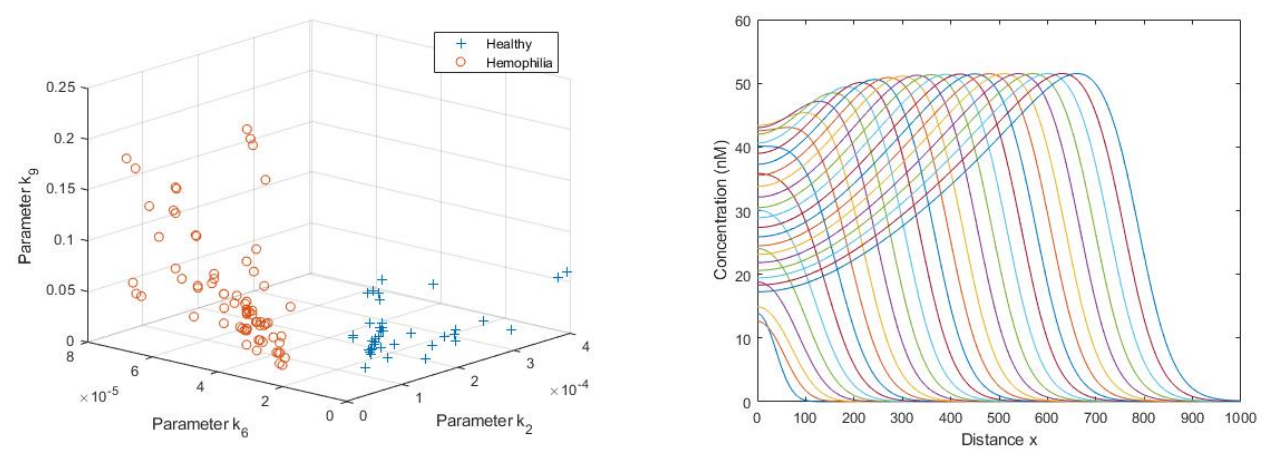

Figure 1. Left: the values of parameters for healthy (crosses) and hemophilia (circles) patients obtained by fitting the experimental thrombin generation curves. Right: wave propagation for a healthy subject $\left(k_{2}=3.030 e^{-4}, k_{3}=1.296 e^{-05}, k_{4}=6.785 e^{-3}, k_{5}=7.205 e^{-06}, k_{6}=1.269 e^{-06}, k_{7}=4.749 e^{-12}, k_{8}=\right.$ $\left.8.172 e^{-12}, k_{9}=1.918 e^{-2}\right)$.

We will use below these values of parameters in order to study spatial clot growth in the healthy and hemophilia cases. The nonlinear PDE system is implemented in MatLab with the PDEPE solver. Time integration uses the ODE15s solver with variable time step and variable order [16-18]. 


\subsection{Wave propagation}

After the initiation at the vessel wall, clot growth represents a self-sustained wave propagation independent of the initiation stage $\left(k_{1}=0\right)$. The latter is replaced by the initial condition for the wave propagation:

$$
T^{0}(x)=\left\{\begin{array}{ll}
T_{0} & \text { if } x<x_{0} \\
0 & \text { if } x \geq x_{0}
\end{array} .\right.
$$

The values of $x_{0}$ and $T_{0}$ are fixed in all simulations. The initials conditions for the other two functions are taken constant: $u_{10}^{0}=0$ and $P^{0}(x)=P_{0}$. An example of wave propagation is shown in Figure 1 (right).

Figure 2 shows the dependence of the wave speed on parameters. The speed increases with the increase of the coefficient $k_{2}$ which determines the rate of production of the factor $X a$. Let us note that for the given initial condition and for considered values of other parameters, the wave propagation is not observed for $k_{2}=0$. Similarly, the wave speed increases with the value of the coefficient $k_{5}$, and it becomes 0 for some positive value. Wave propagation is not observed for lesser values. Since we consider the problem on a positive half-axis, in this case, the wave speed can be negative. Finally, the wave speed decreases with the increase of $k_{9}$. This coefficient shows the intensity of thrombin deactivation by antithrombin.
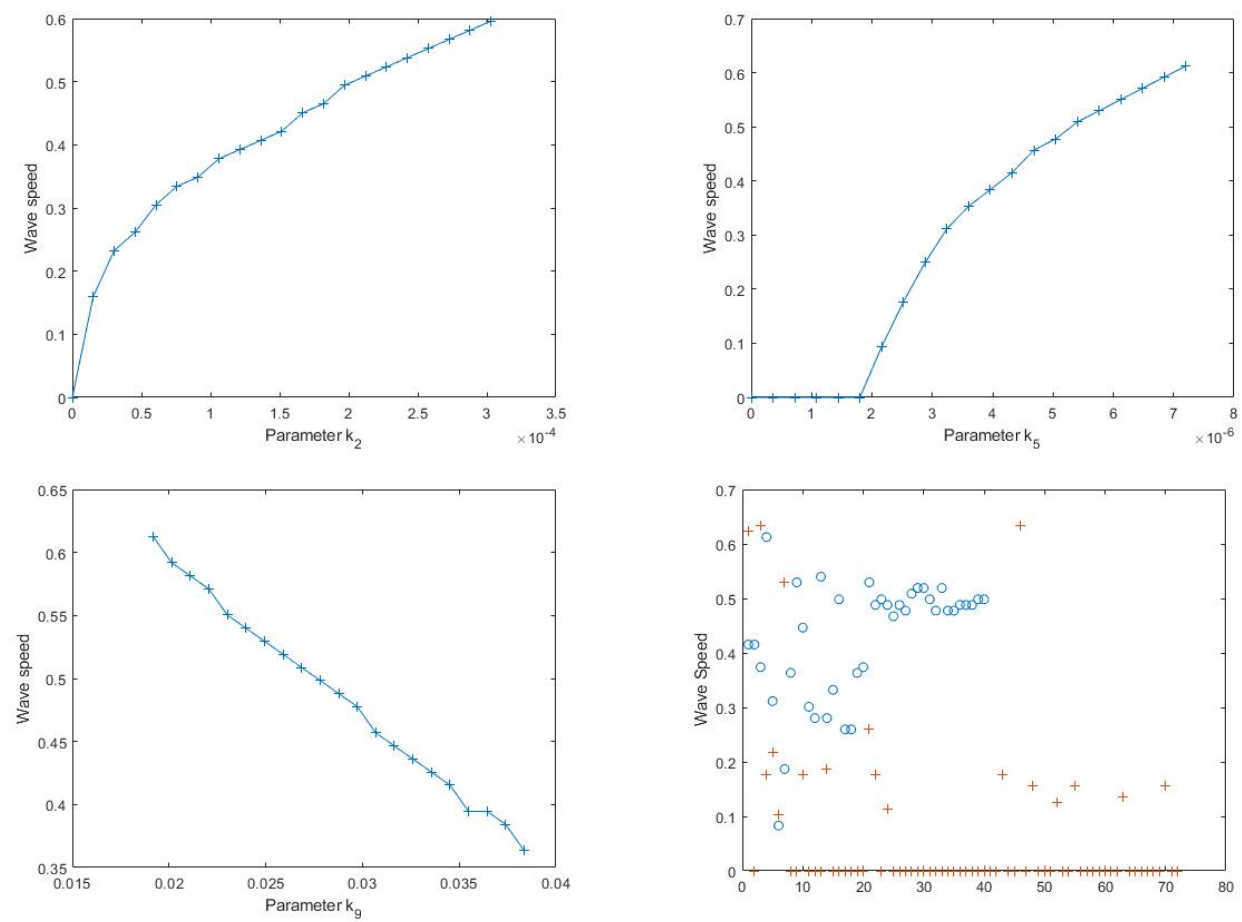

Figure 2. Top figures, and bottom left figure: wave speed depending on parameters $k_{2}, k_{5}$ or $k_{9}$, respectively. Bottom right: wave speed for the values of parameters determined by fitting the experimental data. The $x$-axis shows the number of patient, the $y$-axis the corresponding wave speed; healthy: circles, hemophilia: crosses. 


\subsection{Difference between healthy and hemophilia patients}

Hemophilia patients are characterized by low concentrations of factor VIII or IX. In model (1)-(3), the values of parameters $k_{2}, k_{3}, k_{7}$ and $k_{8}$, depending on these concentrations, are also low. Although parameters $k_{7}$ and $k_{8}$ have a minor impact on the wave speed, parameters $k_{2}$ and $k_{3}$ are very important. Hence, in the majority of cases, for hemophilia patients we observe wave propagation with a low speed, or no propagation at all. Therefore, clot does not form normally, or it does form not at all.

Figure 2 (bottom right) shows that all healthy subjects have positive wave speeds, which leads to normal coagulation. Most of hemophilia subjects have, instead, a low wave speed or no wave propagation, which leads to bleeding disorders. In few cases, the wave speed for hemophilia subjects is similar to the wave speed for healthy subjects. In fact, it has been observed that some hemophilia patients have normal coagulation.

\subsection{The influence of blood flow}

Reaction-diffusion waves describe the propagation phase of clot growth but not growth arrest. Two main mechanisms that stop clot growth are determined by the influence of blood flow and by the activated protein $\mathrm{C}$. We discuss here the first mechanism. Taking into account blood flow, we write system (4) in the following form:

$$
\frac{\partial u}{\partial t}=D \frac{\partial^{2} u}{\partial x^{2}}+F(u)-\sigma(x) u
$$

The last term in the right-hand side of this equation characterizes the influence of the flow velocity on clot growth washing out blood factors from the clot. The function $\sigma(x)$ show the flow velocity as a function of the distance from the wall. In the first approximation, neglecting the influence of the clot on flow velocity, we can consider a Poiseuille profile. For the same values of parameters as in Figure 1 (right) and $\sigma(x)=a x(L-x)$ we obtain the results shown in Figure 3. The clot growth speed decreases with time, and clot stabilizes at some distance from the wall.
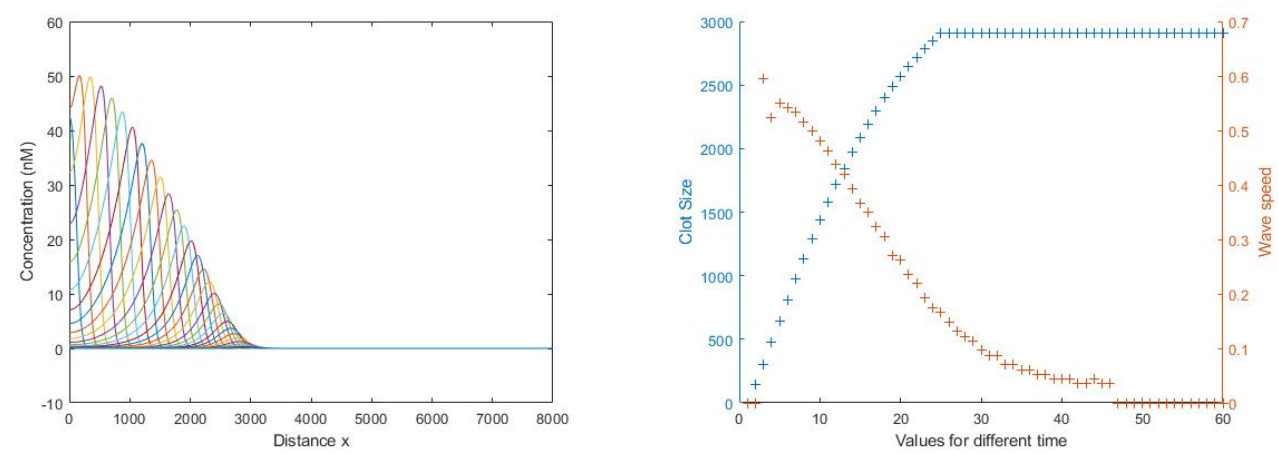

Figure 3. Left: wave propagation for a healthy subjects with a Poiseuille flow $\sigma(x)=a x(L-x)$, where $a=10^{-4} \mu m^{-2} \cdot s^{-1}, L=8 \mu m$. Right: wave speed and clot size as functions of time.

\section{Discussion}

Existence, stability and the speed of propagation of reaction-diffusion waves of blood coagulation determine the properties of clot growth. We have presented in this work some results 
obtained by numerical simulations. The underlying mathematical analysis is usually quite involved. For system (4), these questions are not studied. We will discuss here a simplified system where $k_{9}=0$. Assuming that diffusion coefficients in all equations are equal to each other, we can reduce this system to the system of two equations:

$$
\begin{gathered}
\frac{\partial u}{\partial t}=d \frac{\partial^{2} u_{10}}{\partial x^{2}}+\left(k_{2} T+k_{3} T^{2}\right)\left(v_{10}^{0}-u_{10}\right)-k_{4} u_{10}, \\
\frac{\partial T}{\partial t}=d \frac{\partial^{2} T}{\partial x^{2}}+\left(k_{5} u_{10}+k_{6} T+k_{7} T^{2}+k_{8} T^{3}\right)\left(P_{0}-T\right) .
\end{gathered}
$$

This system has two stationary points: $u_{10}=T=0$ and $T=P_{0}, u_{10}=h v_{10}^{0} /\left(k_{4}+h\right)$, where $h=P_{0}\left(k_{2}+k_{3} P_{0}\right)$. Hence, it is a monostable case, and, since system (7), (8) is monotone, the waves exist for all values of the speed greater than or equal to the minimal speed [19]. These waves are stable, and the minimal speed admits a minimax representation.

Similar results can be expected for small values of the coefficient $k_{9}$. However, if it is sufficiently large, the wave with a positive speed may not exist. Clot growth can be observed for the appropriate choice of initial conditions if the wave exists and its speed is positive. The initial conditions considered above are determined by the values $x_{0}$ and $T_{0}$. They were taken the same for all patients but the result can depend on the choice of these values. In some healthy cases, the wave exists for all nonzero values of $x_{0}$ and $T_{0}$, while for some hemophilia subjects, the wave was never observed. In some intermediate cases, increasing $x_{0}$ or $T_{0}$ supports the convergence to the wave.

Acknkowledgements. V. Volpert was supported by the RUDN University "Program 5100", and the French-Russian project PRC2307.

\section{References}

[1] Hemker, H.C., and S. Béguin, Thrombosis and Haemostasis 74(1), 134-38 (1995).

[2] Hemker, H. C. et al., Pathophysiology of Haemostasis and Thrombosis 32(5-6), 24953(2002).

[3] Hemker, H. Coenraad et al., Pathophysiology of Haemostasis and Thrombosis 33(1), 4-15 (2003).

[4] Lancé, M.D., Thrombosis Journal 13 (2015).

[5] Mann, K.G., S. Butenas, and K. Brummel., Arteriosclerosis, Thrombosis, and Vascular Biology 23(1), 17-25 (2003).

[6] Hoffman, M., Z.H. Meng, H.R. Roberts, and D.M. Monroe, Japanese Journal of Thrombosis and Hemostasis 16(1), 70-81, http://joi.jlc.jst.go.jp/JST.JSTAGE/jjsth/16.70?from=CrossRef (2005).

[7] Chelle, P., C. Morin, A. Montmartin, M. Piot, M. Cournil, and B. Tardy-Poncet, Bull Math Biol 80, 1989-2025 (2018).

[8] Tokarev, A., Yu.V. Krasotkina, M.V. Ovanesov, M.A. Panteleev, M.A. Azhigirova, V.A. Volpert, F.I. Ataullakhanov, A.A. Butilin, Math Model Nat Phenom 1(2), 124-137 (2006).

[9] Panteleev, M.A., M.V. Ovanesov, D.A. Kireev, A.M. Shibeko, E.I. Sinauridze, N.M. Ananyeva, A.A. Butylin, E.L. Saenko and F.I. Ataullakhanov, Biophys J 90, 1489-1500 (2006). 
[10] Panteleev, M.A., A. N. Balandina, E. N. Lipets, M.V. Ovanesov, and F.I. Ataullakhanov, Biophys J 98, 1751-1761 (2010).

[11] Ratto, N., A. Tokarev, P. Chelle, B. Tardy-Poncet, and V. Volpert, Acta Biotheoretica, in press. (2019).

[12] Ataullakhanov, F.I., G.T. Guria, and A.Y. Safroshkina, Biofizika 39(1), 97-106 (1994).

[13] Krasotkina, Y. V., E. I. Sinauridze, and F. I. Ataullakhanov, Biochimica et Biophysica Acta (BBA) - General Subjects 1474(3), 337-345 (2000).

[14] Pogorelova, E. A., and A. I. Lobanov, Biophysics 59(1), 110-118 (2014).

[15] Zarnitsina, V. I., F. I. Ataullakhanov, A. I. Lobanov, and O. L. Morozova, Chaos: An Interdisciplinary Journal of Nonlinear Science 11(1), 57 (2001).

[16] Shampine, L. F. and M. W. Reichelt, SIAM Journal on Scientific Computing 18, 1-22 (1997).

[17] Shampine, L. F., M. W. Reichelt, and J.A. Kierzenka, SIAM Review 41, 538-552 (1999).

[18] Skeel, R. D. and M. Berzins, SIAM Journal on Scientific and Statistical Computing 11, 1-32 (1990).

[19] Volpert, V., Elliptic Partial Differential Equations: Volume 2: Reaction-Diffusion Equations (Birkhauser, Basel, 2014). 